\title{
The Complicated Evolution of the ACIS Contamination Layer over the Mission Life of the Chandra X-ray Observatory
}

\author{
Paul P. Plucinsky ${ }^{\mathrm{a}}$, Akos Bogdan ${ }^{\mathrm{a}}$, Herman L. Marshall ${ }^{\mathrm{b}}$, and Neil W. Tice ${ }^{\mathrm{b}}$ \\ ${ }^{a}$ Harvard-Smithsonian Center for Astrophysics, MS-3, 60 Garden St., Cambridge, MA 02138, \\ USA \\ ${ }^{\text {b} M I T ~ K a v l i ~ I n s t i t u t e ~ f o r ~ A s t r o p h y s i c s ~ a n d ~ S p a c e ~ R e s e a r c h, ~ C a m b r i d g e, ~ M A ~ 02139, ~ U S A ~}$
}

\begin{abstract}
The Chandra X-ray Observatory (CXO) was launched almost 19 years ago and has been delivering spectacular science over the course of its mission. The Advanced CCD Imager Spectrometer (ACIS) is the prime instrument on the satellite, conducting over $90 \%$ of the observations. The CCDs operate at a temperature of $-120 \mathrm{C}$ and the optical blocking filter $(\mathrm{OBF})$ in front of the CCDs is at a temperature of approximately $-60 \mathrm{C}$. The surface of the $\mathrm{OBF}$ has accumulated a layer of contamination over the course of the mission, as it is the coldest surface exposed to the interior to the spacecraft. We have been characterizing the thickness, chemical composition, and spatial distribution of the contamination layer as a function of time over the mission. All three have exhibited significant changes with time. There has been a dramatic decrease in the accumulation rate of the contaminant starting in 2017. The lower accumulation rate may be due to a decrease in the deposition rate or an increase in the vaporization rate or a combination of the two. We show that the current calibration file which models the additional absorption of the contamination layer is significantly overestimating that additional absorption by using the standard model spectrum for the supernova remnant 1E 0102.2-7219 developed by the International Astronomical Consortium for High Energy Calibration (IACHEC). In addition, spectral data from the cluster of galaxies known as Abell 1795 and the Blazar Markarian 421 are used to generate a model of the absorption produced by the contamination layer. The Chandra X-ray Center (CXC) calibration team is preparing a revised calibration file that more accurately represents the complex time dependence of the accumulation rate, the spatial dependence, and the chemical composition of the contaminant. Given the rapid changes in the contamination layer over the past year, future calibration observations at a higher cadence will be necessary to more accurately monitor such changes.
\end{abstract}

Keywords: X-rays, CCDs, Chandra X-ray Observatory, ACIS, contamination

\section{INTRODUCTION}

The Chandra X-ray Observatory (CXO) was launched on 23 July 1999 on the Space Shuttle Columbia. An overview of the mission and its instruments are presented in Weisskopf et al. $(2000)^{1}$ and an update on the mission was provided in Weisskopf et al. (2012). ${ }^{2}$ The CXO carries two imaging instruments, the Advanced CCD Imaging Spectrometer (ACIS) discussed in Garmire et al. $(1992)^{3}$ and Garmire et al. $(2003)^{4}$ and the High Resolution Camera (HRC) discussed in Murray et al. (1997). ${ }^{5}$ In addition, the CXO carries two gratings instruments known as the High Energy Transmission Grating (HETG) described in Canizares et al. $(2000)^{6}$ and the Low Energy Transmissions Grating (LETG) Brinkman et al. (2000). ${ }^{7}$

ACIS is the primary instrument on the CXO with a nominal bandpass of $0.3-10.0 \mathrm{keV}$, conducting over $90 \%$ of the observations. ACIS contains 10 CCDs arranged into two arrays. One array, the ACIS Imaging array (ACIS-I), consists of four frontside illuminated (FI) CCDs arranged in a $2 \times 2$ array, and the other array, the ACIS Spectroscopy array (ACIS-S), consists of four FI CCDs and two backside illuminated (BI) CCDs arranged in a $1 \times 6$ array. The ACIS-I array is used primarily for imaging spectroscopy and the ACIS-S array is used primarily as the readout detector for the HETG and LETG, although the ACIS-S is also used for imaging spectroscopy.

Further author information: (Send correspondence to P.P.P)

P.P.P.: E-mail: pplucinsky@cfa.harvard.edu 
The BI CCDs have higher quantum efficiency at low energies than the FI CCDs and are therefore preferred over the FI CCDs for some imaging observations.

In order to suppress optical to infrared photons but to transmit the X-ray photons of interest, both ACIS arrays have an Optical Blocking Filter (OBF) inserted in the optical path. The filters were produced by Luxe1 ${ }^{\mathrm{TM}}$ and are made of polyimide with $\mathrm{Al}$ deposited on both sides of the polyimide. The two filters are of slightly different thicknesses, the ACIS-S OBF (OBF-S) is 100/200/30 nm of Al/polyimide/Al and the ACIS-I OBF is $130 / 200 / 30 \mathrm{~nm}$ of $\mathrm{Al} /$ polyimide/Al. The OBFs sit about $12 \mathrm{~mm}$ in front of the CCDs facing the mirrors on the CXO. The volume around the CCDs is effectively isolated from the interior of the spacecraft, while the surface of the OBFs facing the mirrors is exposed to the interior of the spacecraft. The CCD focal plane is regulated at a temperature of $-120 \mathrm{C}$. The OBFs are positioned at the top of the ACIS Camera Body (CB) which was regulated at $-60 \mathrm{C}$ early in the mission, but has been unregulated from April 2008 fluctuatng between between $-72 \mathrm{C}$ and -60 C. The CB was regulated at -60 C from August 2015 until July 2016 but has since been unregulated (see Plucinsky et al. $2016^{9}$ for details). In normal operations, the centers of the filters are warmer by $\sim 2-4$ degrees due to the radiative heat load of the warm mirrors $(+20 \mathrm{C})$ and the optical bench assembly.

It was noticed early in the mission ${ }^{8}$ that the low energy sensitivity of the ACIS instrument was decreasing with time. It was quickly determined that this loss of detection efficiency was the result of a contamination layer building up on the surface of the OBFs facing the spacecraft interior. The contamination layer continues to accumulate even after 18 years on orbit. The accumulation rate, the chemical composition, and the spatial distribution of the contaminant have all varied with time over the mission. The accumulation rate exhibited a steep rise at the beginning of the mission, a flattening from 2003 until 2010, and then another steep rise from 2010 onwards. We reported in $2016^{9}$ on our efforts to reduce the accumulation rate by turning on the ACIS Detector Housing (DH) heater which regulates the $\mathrm{CB}$ and hence OBF edges at $-60 \mathrm{C}$. There was no measurable effect on the accumulation rate due to the $\mathrm{DH}$ heater regulating the $\mathrm{CB}$ at $-60 \mathrm{C}$. In this paper we report that the accumulation rate has decreased significantly starting in 2017 and we discuss our current understanding of the time-variable accumulation rate and chemical composition.

\section{ACIS CONTAMINATION LAYER}

\subsection{Discovery and Initial Characterization}

The existence of the contamination layer was discovered in $2002^{8}$ as a gradual decrease in the low energy detection efficiency of all of the CCDs. The growth of the contamination layer was tracked by repeated observations of the external calibration source (ECS) which has lines of Al-K (1.5 keV), Ti-K (4.5 keV), and Mn-K (5.9 keV) from an $\mathrm{Fe}^{55}$ radioactive source with a half-life of $2.7 \mathrm{yr}$. The ECS also produced a line complex from Mn-L around $0.67 \mathrm{keV}$. The ratio of the Mn-L/Mn-K count rates on the S3 BI CCD became the most useful measure of the declining sensitivity at low energies. Unfortunately, the observed flux from the Mn-L line complex decreased with time due to the decay of the radioactive source and the increasing thickness of the contamination layer. Eventually the uncertainties on the measurements became so large that they were no longer useful to track the growth of the contamination layer. As the mission progressed, we transitioned to using celestial sources to monitor the growth of the contamination layer. We used celestial sources that are believed to be constant (or nearly constant on human time scales), such as clusters of galaxies and supernova remnants (SNRs), to monitor the change in low energy detection efficiency. We also used bright, variable sources with the HETG and LETG to constrain the absorption as a function of energy produced by the contaminant.

Early efforts to determine the chemical composition of the contaminant ${ }^{10}$ identified absorption edges of $\mathrm{C}$, $\mathrm{O}$, and $\mathrm{F}$ that were in excess of the edges in the ACIS OBF. The ACIS OBF has absorption edges of $\mathrm{C}$ and $\mathrm{O}$, but no edge due to $\mathrm{F}$. The ACIS detection efficiency as a function of energy was carefully calibrated before launch ${ }^{11,12}$ including the transmission and absorption edges of the OBFs. The flight measurements used a bright continuum source dispersed with the HETG and/or LETG to achieve the highest spectral resolution possible with the CXO. In these high resolution spectra, it became obvious that some absorption edges were deeper than in the pre-flight measurements or only appeared (in the case of $\mathrm{F}$ ) after launch. The newly-detected absorption edges were also found to be increasing in time. $\mathrm{C}$ was by far the dominant species in the contaminant while the $\mathrm{O}$ and $\mathrm{F}$ were approximately equal in concentration. We believe the contaminant started accumulating as soon 


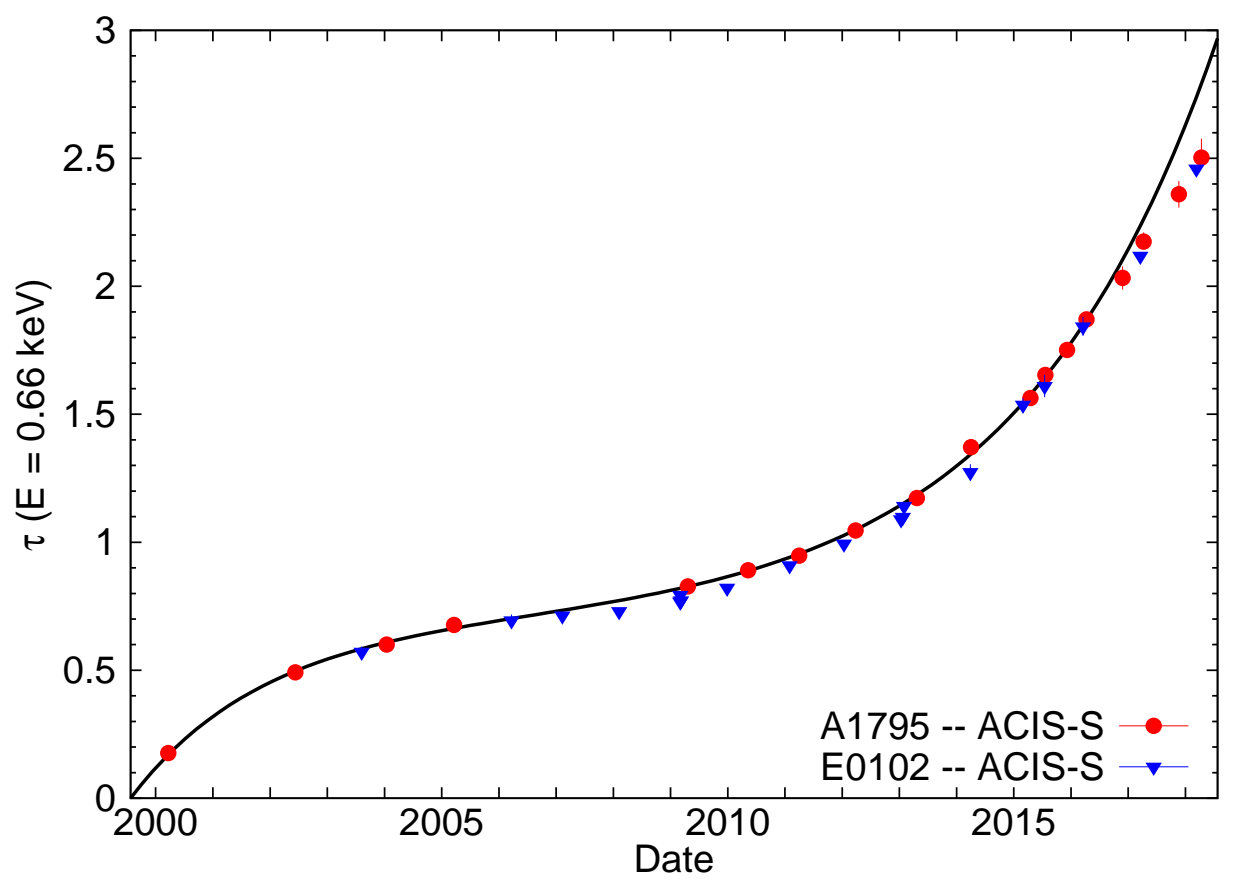

Figure 1. Optical depth at $0.67 \mathrm{keV}$ for the ACIS-S aimpoint as determined by fits to the E0102(blue) and A1795(red) data. The black line is the model for the optical depth in the N0010 contamination model.

as the ACIS door was opened and the OBFs were exposed to the interior of the spacecraft. The contaminant has continued to accumulate for the entire 19 year mission of the CXO, see Section 2.3 for a detailed time history.

\subsection{OBF and Camera Body Temperatures}

ACIS has two separate filters, one for the Imaging array, OBF-I, and one for the spectroscopy array, OBF-S. For diagrams and pictures of the flight hardware, see the figures in Plucinsky et al. (2004). ${ }^{13}$ Both OBFs are secured to the top surface of the ACIS Camera Body (CB). The OBFs have no active thermal control but respond to the environment around them. The edges of the filter are in good thermal contact with the $\mathrm{CB}$ and are therefore at the same temperature as the $\mathrm{CB}$. At the beginning of the mission, the $\mathrm{CB}$ was held at $-60 \mathrm{C}$. The centers of the filter are warmer than the edges due to the radiative heat load from the warm mirrors and optical bench cavity. The center of the OBF-I is modeled to achieve a temperature of $\sim-56 \mathrm{C}$ while the center of the OBF-S is at about $\sim-58 \mathrm{C}$. There is no temperature sensor on the OBFs themselves.

In April 2008, it was decided to turn off the ACIS Detector Housing (DH) heater which kept the CB temperature at -60 C. With the DH heater off, the CB temperature fluctuated between $-72 \mathrm{C}$ and $-62.5 \mathrm{C}$ depending on the orientation of the CXO spacecraft. The cooler CB temperature provided more margin for keeping the CCDs in the focal plane at -120 C. From launch in 1999 until April 2008, the CB temperature regulated at -59.9 C except for a few excursions during special activities. After April 2008, the CB temperature was unregulated and varied with the orientation of the spacecraft. In August 2015, it was decided to turn the ACIS DH heater back on with the hope that the accumulation rate of the contaminant would decrease. But as we reported in $2016,{ }^{9}$ the warmer CB temperatures has no effect on the accumulation rate of the contaminant. Therefore, it was decided in July 2016 to turn the DH heater back off and leave the CB temperatures unregulated.

\subsection{Time Dependence of the Accumulation Rate}

As mentioned in Section 2.1, the accumulation rate of the contamination layer was monitored with the ECS until the radioactive source became too faint to produce reliable results. At this point, we switched to using the brightest SNR in the Small Magellanic Cloud, 1E 0102.2-7219 (hereafter E0102), a bright cluster of galaxies 


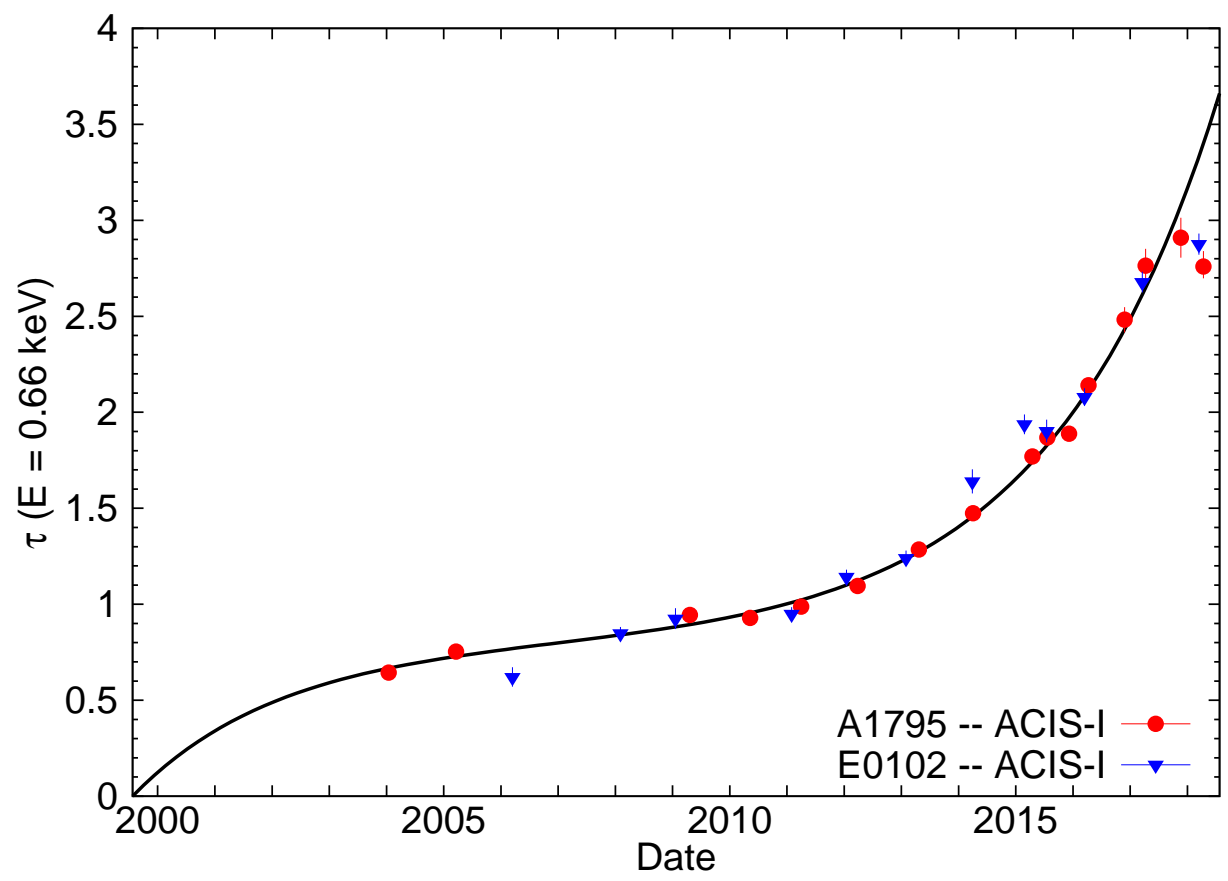

Figure 2. Optical depth at $0.67 \mathrm{keV}$ for the ACIS-I aimpoint as determined by fits to the E0102(blue) and A1795(red) data. The black line is the model for the optical depth in the N0010 contamination model.

known as Abell 1795 (hereafter A1795), and a bright Blazar called Markarian 421 (hereafter Mkn 421). E0102 has a soft, line-dominated spectrum and we have used it throughout the mission to characterize the contamination layer. ${ }^{14,15}$ The development of the standard IACHEC model for E0102 and its application to the current generation of X-ray instruments is presented in our 2017 paper. ${ }^{16}$ A1795 has a harder thermal spectrum with some significant line emission. Mkn 421 has a continuum spectrum described by a curved power-law model.

We have used the A1795 and E0102 data on ACIS-S and ACIS-I to measure the optical depth of the contaminant at $0.67 \mathrm{keV}$ (the energy of the Mn-L complex in the ECS). The results for the ACIS-S aimpoint are plotted in Figure 1. The blue data points are derived from the E0102 data, the red data points are derived from the A1795 data and the black curve shows the expected increase in the contamination layer that is contained in the current release of the CXC contamination file "acisD1999-08-13contamN0010.fits", called "N0010" for short. The measured optical depths from the E0102 and A1795 data are consistent within the uncertainties. The accumulation history of the contaminant is shown in this figure, a steep rise early in the mission, a reduction in the rate from 2003 to 2010, another sharp increase after 2010, and an apparent decrease starting in 2017. The data in 2017 begin to deviate from the expected accumulation rate and the trend continues into 2018. The decrease in the accumulation rate is not correlated with the DH heater which was on from 11 August 2015 until 20 July 2016. The behavior at the aimpoint on ACIS-I is even more dramatic and shown in Figure 2. The first data point to deviate from the expectation is in late 2017. Perhaps more interesting, the last data point in 2018 is consistent with no accumulation over the last 6 months. The uncertainties are relatively large so future measurements will be necessary to confirm this result. Note that the maximum optical depth is about 3.0 on the OBF-I and is about 2.5 on the OBF-S. The contaminant has apparently accumulated more rapidly at the center of the OBF-I than at the center of the OBF-S. This can be seen more clearly in Figure 3 which shows the difference in the optical depth at the aimpoints on ACIS-I and ACIS-S as a function of time. For most of the mission, the optical depths were within 0.2 of each other. But starting in 2015, the contaminant grew more rapidly near the aimpoint on ACIS-I reaching a maximum difference of 0.6 optical depths. Curiously, the most recent data point in 2018 shows the difference between OBF-I and OBF-S is decreasing. This suggests that the accumulation rate on OBF-I is close to zero while the accumulation rate is still positive and small on OBF-S. Future observations will be necessary to determine if this trend will continue. 


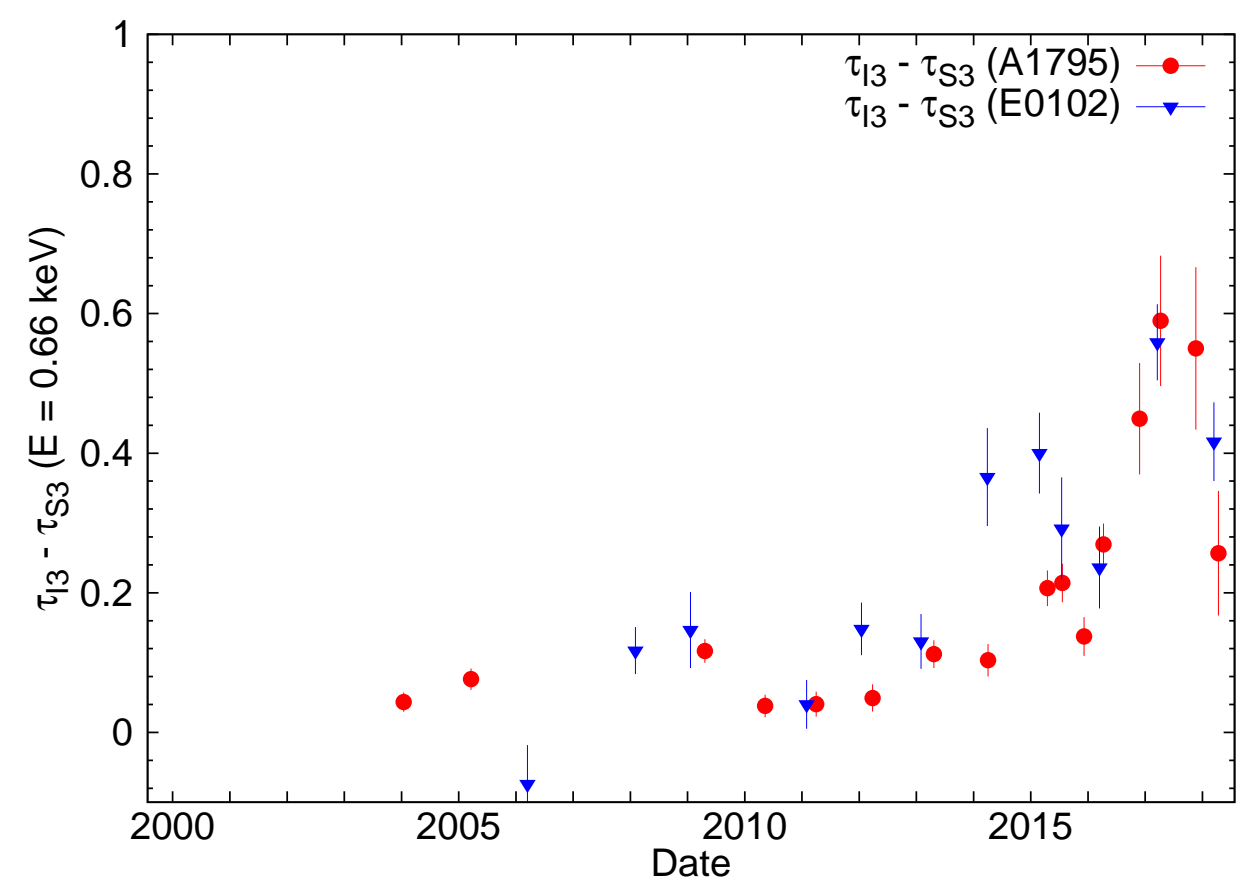

Figure 3. The difference in the optical depth at $0.67 \mathrm{keV}$ at the ACIS-I and ACIS-S aimpoints. The contaminant grew more quickly on OBF-I than OBF-S from 2015 until 2017.

\subsection{Time Dependence of the Chemical Composition}

The high resolution spectra provided by the HETG have been used ${ }^{10}$ to constrain the chemical composition of the contaminant and how it has changed with time. The contaminant is composed mostly of $\mathrm{C}$, with some $\mathrm{O}$ and F. One limitation of the HETG data is that they only provide information on the contaminant for the OBF-S filter. The optical depth of the contaminant for each element $(\mathrm{C}, \mathrm{O}, \& \mathrm{~F})$ is modeled as a functions of time and position with a two component model:

$$
\tau(t ; x, y)=\tau_{0}(t)+\left[\tau_{1}(t) \times f(x, y)\right]
$$

where $\tau_{0}(t)$ represents a time-variable, spatially uniform component, $\tau_{1}(t)$ represents a time-variable, spatially variable component, and $f(x, y)$ is the spatial distribution for the spatially variable component. Figure 4 shows the time dependence of the $\tau_{0}(t)$ and $\tau_{1}(t)$ components for $\mathrm{C}$ near the aimpoint on the ACIS-S detector derived from HETG observations of Mkn 421. The time dependence of $\tau_{0}(t)$ for $\mathrm{C}$ matches that of the N0010 model until the last few data points which are significantly below the line. This is similar to the behavior seen for A1795 and E0102 shown in Figure 1. The time dependence of $\tau_{1}(t)$ for $\mathrm{C}$ matches that of the N0010 model in shape, but the N0010 model might be slightly under-predicting at late times. The $\tau_{1}(t)$ component has been mostly flat with time from 2015 onwards, while the $\tau_{0}(t)$ continues to accumulate, albeit at a lower rate than predicted by the N0010 model. One interpretation of this behavior is that the spatially uniform component and the spatially variable component correspond to separate materials and the spatially variable component has ceased to accumulate.

Figure 5 shows the time dependence $\tau_{0}(t)$ and $\tau_{1}(t)$ components for $\mathrm{O}$, again near the aimpoint on the ACIS-S detector. The time dependence of $\tau_{0}(t)$ for $\mathrm{O}$ matches that of the N0010 model until the last few data points which are significantly above the line. The time dependence of $\tau_{1}(t)$ for $\mathrm{O}$ matches that of the N0010 model in shape and amplitude. However, the data since 2015 are consistent with no growth in this component so the N0010 model may be over-predicting the contaminant at late times but the uncertainties are still large enough that the case is not definitive. The $\tau_{0}(t)$ result indicates that the N0010 model has less $\mathrm{O}$ than it should. But 

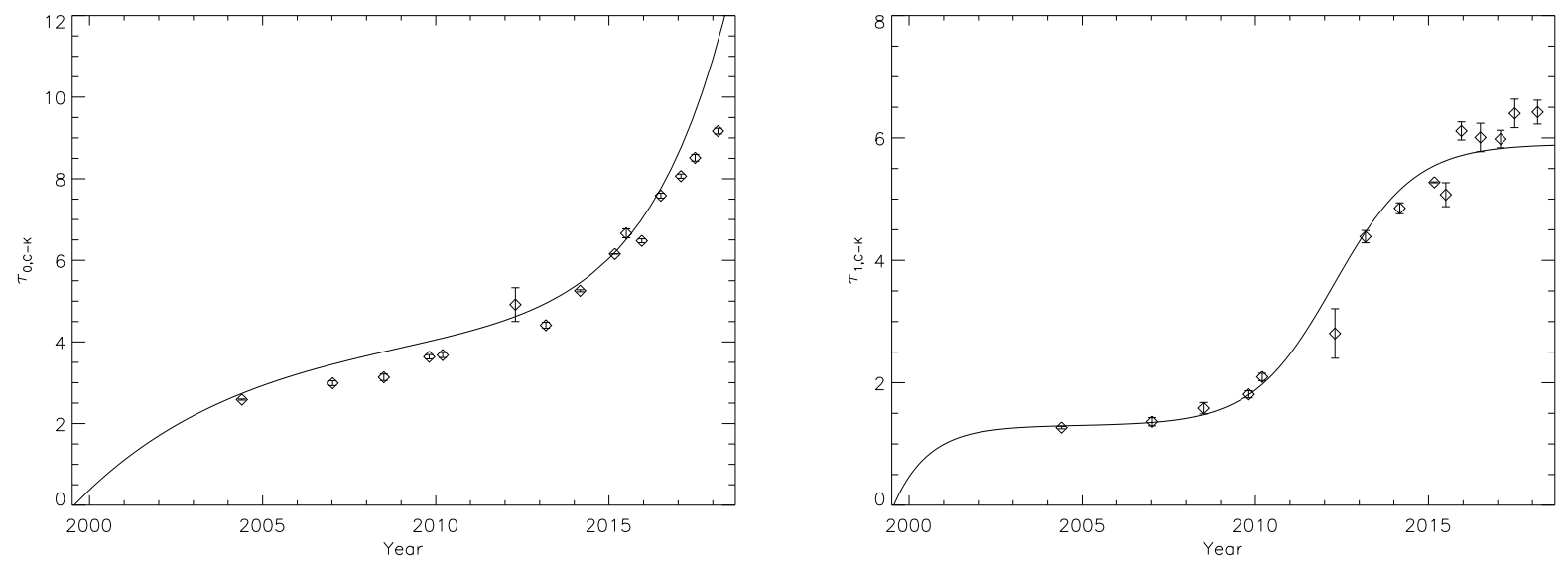

Figure 4. LEFT: The optical depth at C-K of the spatially uniform component near the center of the ACIS-S array. RIGHT: The optical depth at C-K of the spatially variable component near the center of the ACIS-S array. The solid line for both is the prediction from the N0010 contamination model.
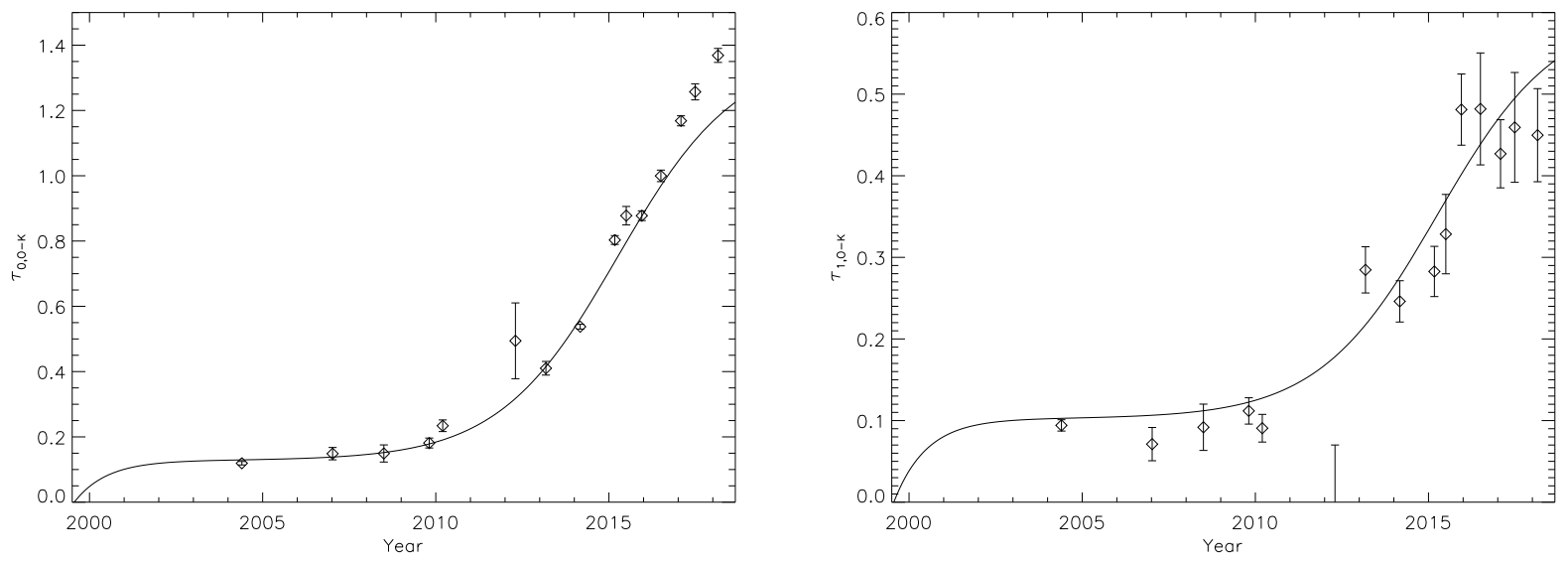

Figure 5. LEFT: The optical depth at O-K of the spatially uniform component near the center of the ACIS-S array. RIGHT: The optical depth at O-K of the spatially variable component near the center of the ACIS-S array. The solid line for both is the prediction from the N0010 contamination model.

note that the total optical depth of $\mathrm{O}$ is significantly less than that of $\mathrm{C}, \sim 2.0$ versus $\sim 15$, so that any error in the $\mathrm{O}$ optical depth has less effect on the observed spectra and is therefore more difficult to discern.

\section{PERFORMANCE OF THE CURRENT CONTAMINATION FILE}

\subsection{Calibration Files}

The CXC calibration group is responsible for providing calibration files that accurately model the additional absorption produced by the contamination layer. As mentioned above the characterization of the contamination layer is complicated by the temporal variation of the thickness, the chemical composition and the spatial distribution. The CXC regularly acquires calibration data of standard targets such as E0102, A1795, and Mkn 421 to verify the current contamination calibration file. If deficiencies are found, a new calibration file is created to address those deficiencies. The ACIS contamination file has been updated 7 times over the course of the CXO mission. For the analysis that follows, we use version N0010 of the model, which is called acisD1999-08-13contamN0010.fits in the CXC Calibration Database (CALDB). We used Chandra Interactive Analysis of Observations (CIAO) version 4.9 and CALDB version 4.7.8. 


\subsection{E0102 Model}

We have defined a standard model for E0102 as part of the activities of the IACHEC. We have used this model extensively ${ }^{14-16}$ to test and improve the ACIS response model earlier in the mission. The model is intended for calibration analyses and is not intended to provide any insight into E0102 as a SNR. The model is empirical in that it uses 52 Gaussians to model the line emission. It uses a two component absorption model, one component for the Galactic contribution and one for the Small Magellanic Cloud (SMC) contribution. We modeled the continuum using a modified version of the Astrophysical Plasma Emission Code (APEC) ${ }^{17}$ called the " No-Line" ' model. This model excludes all line emission, while retaining all continuum processes including bremsstrahlung, radiative recombination continua (RRC), and the two-photon continuum from hydrogenic and helium-like ions (from the strictly forbidden ${ }^{2} S_{1 / 2} 2 s \rightarrow$ gnd and ${ }^{1} S_{0} 1 s 2 s \rightarrow$ gnd transitions, respectively). We included two continuum components of this type in the E0102 model. For details of the model and the parameters assumed see Plucinsky et al. (2017). ${ }^{16}$

Table 1. ACIS S3 Observations of E0102

\begin{tabular}{|c|c|c|c|c|c|c|c|c|c|}
\hline ObsID & Date & ChipX & ChipY & Node & $\begin{array}{r}\text { Exposure } \\
(\mathrm{s}) \\
\end{array}$ & $\begin{array}{r}\text { Counts } \\
(0.3-2 \mathrm{keV})\end{array}$ & $\begin{array}{c}\text { Frame } \\
(\mathrm{s})\end{array}$ & 1stRow & Nrows \\
\hline 3545 & 2003.60 & 357.6 & 496.8 & 1 & 7863.8 & 57133 & 1.1 & 385 & 256 \\
\hline 6765 & 2006.22 & 106.8 & 498.8 & 0 & 7636.7 & 51768 & 0.8 & 384 & 256 \\
\hline 8365 & 2007.11 & 100.2 & 494.3 & 0 & 20985.3 & 138698 & 0.8 & 384 & 256 \\
\hline 9694 & 2008.10 & 100.1 & 492.8 & 0 & 19196.5 & 124804 & 0.8 & 384 & 256 \\
\hline 10654 & 2009.17 & 98.9 & 495.2 & 0 & 7307.1 & 45532 & 0.8 & 335 & 256 \\
\hline 10655 & 2009.17 & 98.2 & 493.0 & 0 & 6810.7 & 43234 & 0.4 & 433 & 128 \\
\hline 10656 & 2009.18 & 342.0 & 494.3 & 1 & 7763.9 & 48603 & 0.8 & 335 & 256 \\
\hline 11957 & 2009.99 & 103.2 & 491.1 & 0 & 18447.8 & 112437 & 0.8 & 335 & 256 \\
\hline 13093 & 2011.08 & 89.8 & 487.9 & 0 & 19049.4 & 108275 & 0.8 & 335 & 256 \\
\hline 14258 & 2012.03 & 86.1 & 493.7 & 0 & 19049.3 & 102049 & 0.8 & 360 & 256 \\
\hline 15555 & 2013.03 & 676.5 & 489.5 & 2 & 23837.3 & 114679 & 0.8 & 360 & 256 \\
\hline 15558 & 2013.06 & 854.4 & 479.7 & 3 & 23051.7 & 107679 & 0.8 & 360 & 256 \\
\hline 15556 & 2013.07 & 666.1 & 164.2 & 2 & 23841.3 & 94733 & 0.8 & 42 & 256 \\
\hline 15467 & 2013.08 & 91.7 & 488.9 & 0 & 19082.2 & 92610 & 0.8 & 360 & 256 \\
\hline 15557 & 2013.09 & 657.9 & 917.2 & 2 & 24191.7 & 78972 & 0.8 & 769 & 256 \\
\hline 15559 & 2013.09 & 854.8 & 162.4 & 3 & 23842.1 & 92216 & 0.8 & 42 & 256 \\
\hline 16589 & 2014.24 & 78.1 & 485.5 & 0 & 9569.5 & 40194 & 0.8 & 360 & 256 \\
\hline 17380 & 2015.16 & 119.2 & 489.0 & 0 & 17655.1 & 65808 & 0.8 & 360 & 256 \\
\hline 17381 & 2015.18 & 659.7 & 165.6 & 2 & 9573.6 & 27095 & 0.8 & 42 & 256 \\
\hline 17382 & 2015.18 & 671.5 & 926.7 & 2 & 9572.7 & 21161 & 0.8 & 769 & 256 \\
\hline 17688 & 2015.54 & 110.7 & 481.6 & 0 & 9569.6 & 33973 & 0.8 & 360 & 256 \\
\hline 17689 & 2015.54 & 661.3 & 158.0 & 2 & 9573.5 & 25482 & 0.8 & 42 & 256 \\
\hline 17690 & 2015.54 & 660.8 & 914.2 & 2 & 9572.7 & 20772 & 0.8 & 769 & 256 \\
\hline 18418 & 2016.21 & 99.0 & 480.2 & 0 & 14326.2 & 45687 & 0.8 & 360 & 256 \\
\hline 18420 & 2016.20 & 651.9 & 919.2 & 2 & 19085.4 & 36556 & 0.8 & 769 & 256 \\
\hline 18419 & 2016.22 & 642.3 & 157.9 & 2 & 19084.7 & 45491 & 0.8 & 42 & 256 \\
\hline 19850 & 2017.22 & 98.6 & 484.9 & 0 & 14326.21 & 38782 & 0.8 & 360 & 256 \\
\hline 19851 & 2017.23 & 647.6 & 166.6 & 2 & 19085.35 & 38603 & 0.8 & 42 & 256 \\
\hline 19852 & 2017.24 & 652.3 & 922.4 & 2 & 19085.38 & 30461 & 0.8 & 769 & 256 \\
\hline 20639 & 2018.20 & 103.8 & 489.4 & 0 & 14326.21 & 32681 & 0.8 & 360 & 256 \\
\hline 20640 & 2018.12 & 645.2 & 165.3 & 2 & 19013.47 & 32687 & 0.8 & 42 & 256 \\
\hline 20641 & 2018.10 & 658.2 & 923.1 & 2 & 19084.61 & 26226 & 0.8 & 769 & 256 \\
\hline
\end{tabular}

Although the standard IACHEC model has many parameters, most of them are held fixed when we fit the data for calibration purposes. The continuum components are fixed and the interstellar absorption components 


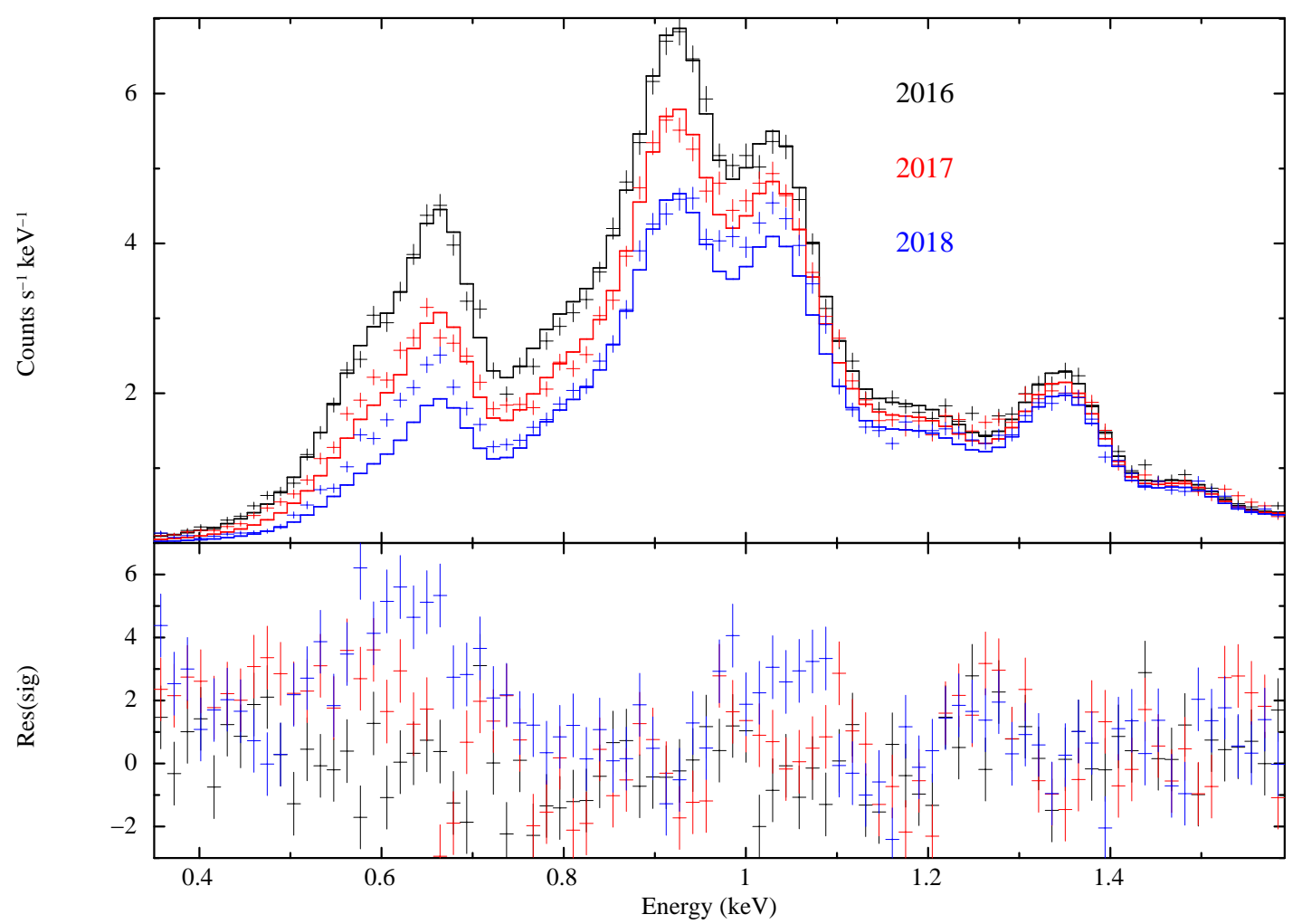

Figure 6. ACIS-S3 spectra of E0102 from OBSIDs 18418(2016), 19850(2017) and 20639(2018). The 2016 data are fit with the standard IACHEC model and that model is overplotted on the 2017 and 2018 data.

are held fixed. All the line energies and widths are also held fixed. Typically, we freeze all line normalizations except for the four normalizations of the brightest lines/line complexes. We allow the normalizations for the O viI He $\alpha r$ line, the O viII Ly $\alpha$ line, the Ne IX He $\alpha r$ line, and Nex Ly $\alpha$ line to vary in the fit. For the O viI He $\alpha$ and Ne IX He $\alpha$ triplets, we link the normalizations of the $f, i$, and $r$ lines to each other and only allow one of them to vary during the fitting process. In this way, the triplet can increase or decrease its normalization as a group but the normalizations of the individual lines in the triplet can not vary independently of each other. There is also a global constant that multiplies the entire spectrum that is allowed to vary. In this manner, we allow only 5 of the 208 parameters in the IACHEC model to vary when we fit. We are essentially allowing the normalizations of the four brightest line/line complexes to vary while freezing the weaker lines and the continuum. We assume that E0102 is not changing significantly over the 19 year lifetime of the CXO mission such that the flux from the source in 1999 is not significantly different from the flux in 2018. And therefore we assume the total flux in a given line is not changing or changing very little over the 19 year mission.

\subsection{ACIS-S Results}

E0102 has been observed many times with ACIS-S since the beginning of the mission. The mirrors on the CXO mission produce such sharp X-ray images that observations of a point source can be affected by "pileup". "Pileup" is defined as two photons interacting with the CCD within one detection cell (typically a $3 \times 3$ pixel region) within one readout frame of the CCD. Even though E0102 is an extended source, some of the bright filaments in E0102 are bright enough to have significant pileup. Most of the observations of E0102 early in the mission were executed in "full-frame" mode with an integration time of $3.2 \mathrm{~s}$. We have excluded the "full-frame" observations from our analysis and selected only the "subarray" observations with shorter frametimes of $1.1 \mathrm{~s}$, $0.8 \mathrm{~s}$, and $0.4 \mathrm{~s}$ in order to minimize the effects of pileup on our data. There are 32 subarray observations of E0102 on S3 included in our analysis listed in Table 1. Most of these observations are near the center of the CCD with chipy values around 512 , but 13 of the observations are at different chipy positions. 


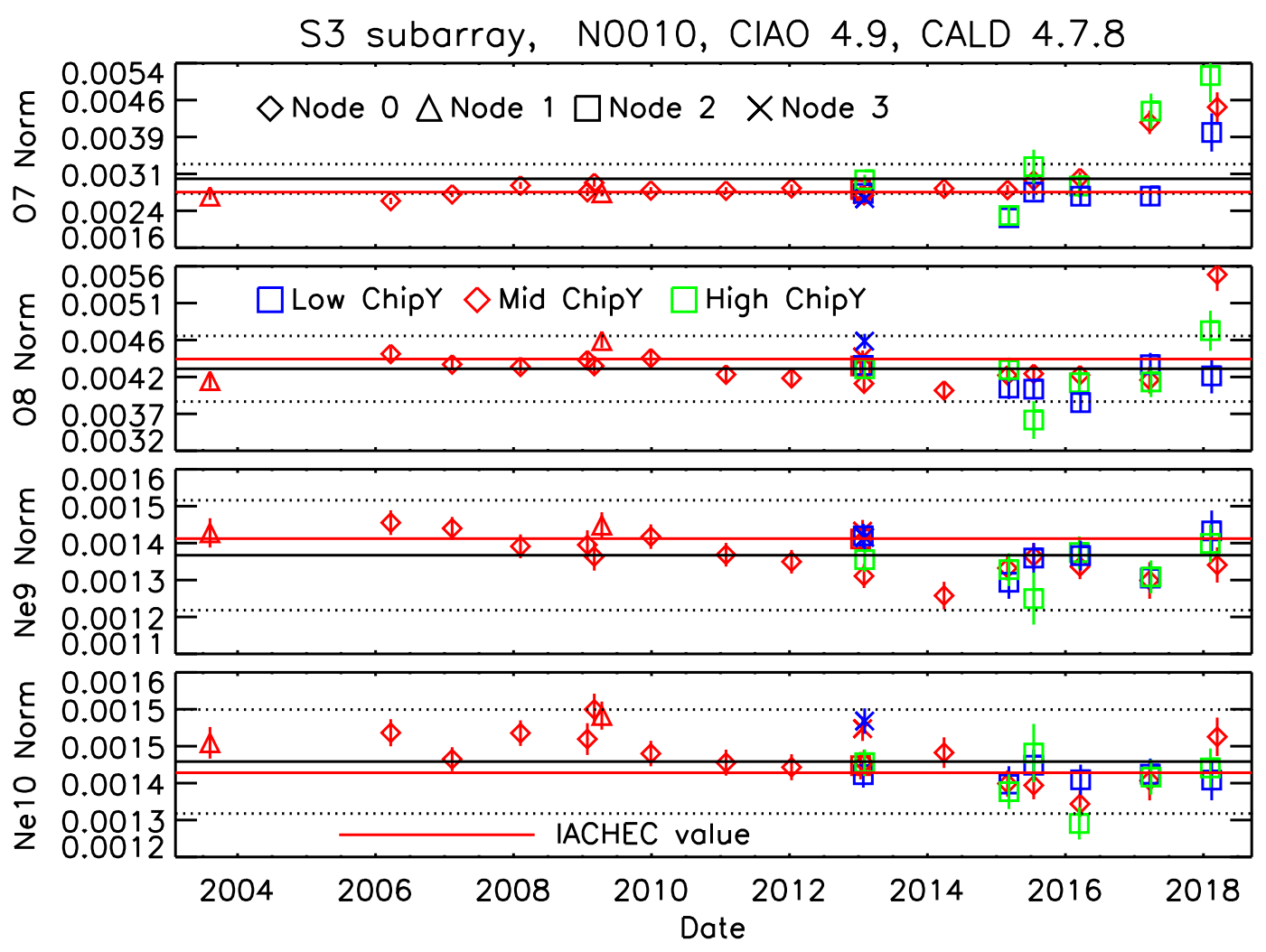

Figure 7. Line normalizations from E0102 on S3 as a function of time. The solid black line is the average of the data points near the on-axis point aimpoint. The red dashed lines are $+/-10 \%$ above and below the average. The points away from the nominal aimpoint are indicated in green and blue.

We have fit all of the S3 observations with the standard IACHEC model allowing only the global normalization and the normalizations for the O VII He $\alpha r$ line, the O viII Ly $\alpha$ line, the Ne IX He $\alpha r$ line, and Nex Ly $\alpha$ line to vary. Figure 6 shows an example of these fits for the three most recent observations near the S3 aimpoint from 2016, 2017 and 2018. The model was fit to the 2016 data and then frozen for the 2017 and 2018 observations to demonstrate deficiencies in the time-dependent contamination model. The large residuals in the 2018 spectrum at the $\mathrm{O}$ vIII Ly $\alpha$ and Nex Ly $\alpha$ lines indicate that the contaminant is over-estimated. Note that the difference between the 2017 and 2018 observations is not as large as the model predicts. The residuals indicate that the O viII Ly $\alpha$ line and the Nex Ly $\alpha$ are not well fitted in 2018 but the Ne IX He $\alpha r$ line is well fitted. This will be challenging to correct with a revised contamination model.

We compared the fitted line normalizations for the O VII He $\alpha r$ line, the O viII Ly $\alpha$ line, the Ne IX He $\alpha r$ line, and the Nex Ly $\alpha$ line as a function of time. The results are plotted in Figure 7. The solid black line is the average of the on-axis data and the black dashed lines are $+/-10 \%$ from the average. Figure 7 shows that the line normalizations are mostly consistent to within $\pm 10 \%$ from 2003 through 2016 for the on-axis data points with the exception of the O VII He $\alpha r$ line. After 2016, the O VII He $\alpha r$ line and O VIII Ly $\alpha$ deviate dramatically from the previous values. The 2018 normalizations on-axis for the O viII Ly $\alpha$ line and O vir He $\alpha r$ line are $\sim 28 \%$ and $\sim 49 \%$ higher than the average value. The NeIX He $\alpha r$ line, and Nex Ly $\alpha$ line normalizations are consistent with the average within $10 \%$ so the problem on S3 appears to effect energies below $0.9 \mathrm{keV}$.

\subsection{ACIS-I Results}

There are 16 subarray observations of E0102 on the I3 CCD in the ACIS-I array. Table 2 lists the observations with their locations on the CCD and exposure times and count rates. Unlike the S3 CCD where the aimpoint is near the middle of the CCD, the aimpoint on the I3 CCD is near the top, right corner (high chipx and chipy). 
Hence most of these observations have chipx of $\sim 875$ and chipy values of $\sim 930$. This position is close to the center of the OBF-I filter, so the contamination layer is thinner at this position than near the edges. There are only 3 of the 14 observations that are at positions other than the nominal aimpoint.

Table 2. ACIS I3 Observations of E0102

\begin{tabular}{rcccrrrrrr}
\hline ObsID & Date & ChipX & ChipY & $\begin{array}{r}\text { Node } \\
\text { Exposure } \\
(\mathrm{s})\end{array}$ & $\begin{array}{r}\text { Counts } \\
(0.3-2 \mathrm{keV})\end{array}$ & $\begin{array}{r}\text { Frame } \\
(\mathrm{s})\end{array}$ & 1stRow & Nrows \\
\hline 3526 & 2003.59 & 884.6 & 120.8 & 3 & 7859.9 & 21368 & 1.0 & 1 & 256 \\
6756 & 2006.20 & 888.7 & 922.5 & 3 & 7159.9 & 23197 & 0.8 & 768 & 256 \\
9690 & 2008.09 & 884.4 & 925.6 & 3 & 19192.5 & 60496 & 0.8 & 768 & 256 \\
10649 & 2009.05 & 880.3 & 926.4 & 3 & 7637.5 & 23510 & 0.8 & 768 & 256 \\
11956 & 2009.98 & 882.6 & 922.3 & 3 & 19189.4 & 57787 & 0.8 & 768 & 256 \\
13092 & 2011.08 & 875.1 & 940.0 & 3 & 19050.1 & 53984 & 0.8 & 768 & 256 \\
14257 & 2012.04 & 881.9 & 928.7 & 3 & 19051.0 & 51539 & 0.8 & 768 & 256 \\
15466 & 2013.08 & 875.5 & 937.8 & 3 & 19082.9 & 47984 & 0.8 & 768 & 256 \\
15471 & 2013.09 & 877.4 & 505.8 & 3 & 9574.3 & 22301 & 0.8 & 341 & 256 \\
15472 & 2013.09 & 873.9 & 144.6 & 3 & 9574.3 & 16405 & 0.8 & 1 & 256 \\
16588 & 2014.24 & 875.4 & 953.9 & 3 & 9571.9 & 21634 & 0.8 & 768 & 256 \\
17379 & 2015.15 & 872.9 & 929.4 & 3 & 17616.6 & 35717 & 0.8 & 768 & 256 \\
17687 & 2015.54 & 866.5 & 934.0 & 3 & 13285.4 & 25598 & 0.8 & 768 & 256 \\
18417 & 2016.20 & 870.5 & 938.8 & 3 & 22886.1 & 40565 & 0.8 & 768 & 256 \\
19849 & 2017.21 & 877.0 & 938.6 & 3 & 23837.4 & 36761 & 0.8 & 768 & 256 \\
20638 & 2018.20 & 877.7 & 933.7 & 3 & 23838.1 & 32164 & 0.8 & 768 & 256 \\
\hline
\end{tabular}

Figure 8 shows an example of these fits for the three most recent observations near the I3 aimpoint from 2016, 2017 and 2018. Again, the model was fit to the 2016 data and then frozen for the 2017 and 2018 data to demonstrate deficiencies in the time-dependent contamination model. Note the dramatic difference in the expected model spectrum for the 2018 data. The N0010 contamination model is over-estimating the contamination by a large amount at the aimpoint on I3. This is partly due to the fact that the accumulation rate has decreased but it is also due to the fact that the N0010 contamination model predicted significantly more contamination at the aimpoint on I3 than S3 (see Figures 1 and 2).

We compared the fitted line normalizations for the O vil He $\alpha r$ line, the O VIII Ly $\alpha$ line, the Ne IX He $\alpha r$ line, and the Nex Ly $\alpha$ line as a function of time. The results are plotted in Figure 9. The over-correction for the contamination layer in 2018 is large. The normalizations for the O VII He $\alpha r$ line, the O vIII Ly $\alpha$ line, the Ne IX He $\alpha r$ line, and the Nex Ly $\alpha$ line are over-estimated by $\sim 98 \%, \sim 125 \%, \sim 32 \%$, and $\sim 25 \%$ respectively. The data from 2016 and earlier are mostly consistent with each other to within $10 \%$. The discrepancy begins in 2017 and dramatically worsens in 2018. The revised contamination file soon to be released by the CXC should address most of this dicrepancy.

\section{POSSIBLE EXPLANATIONS FOR THE REDUCTION IN THE ACCUMULATION RATE}

The analyses presented up to this point measure the accumulation rate of the contaminant which is the difference between the deposition rate and the vaporization rate. If the accumulation changes, we do not know if the deposition rate changed or the vaporization rate changed or both. Over the course of the mission, many components on the CXO spacecraft have increased in temperature, reaching mission high values within the last few years. It is conceiveable that a component on the spacecraft was not out-gassing significantly early in the mission, but as its temperature increased it began to out-gas at a higher rate. Perhaps the out-gassing from this component has now started to decrease, as the source of the contaminant has diminished. Another possibility is that the temperature distributions on the filters have changed with time. Figure 10 shows the expected temperature distributions on the filters in the presence of no contamination when the emittance is expected to 


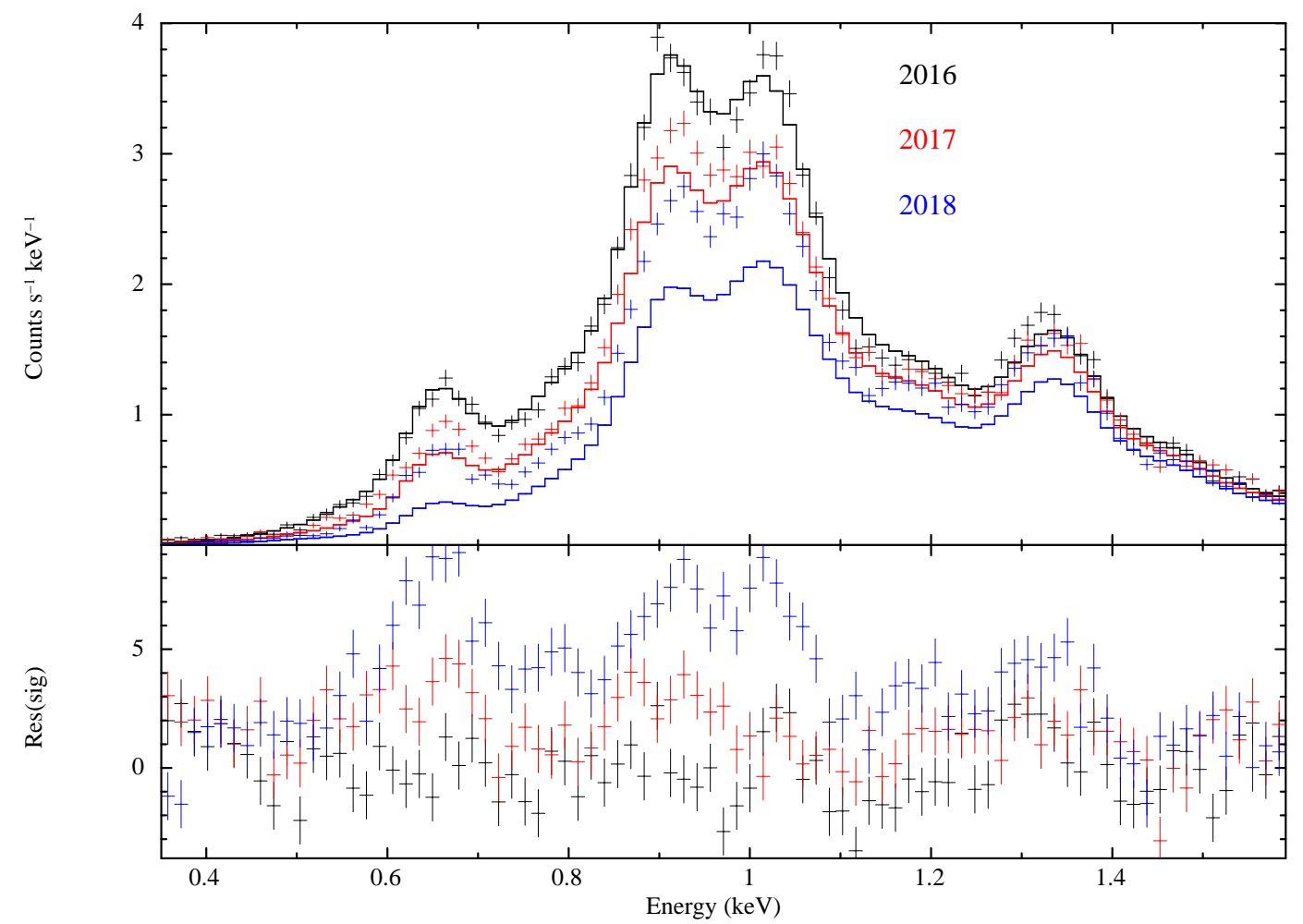

Figure 8. ACIS-I3 spectra of E0102 from OBSIDs 18417(2016), 19849 (2017) and 20638(2018). The 2016 data are fit with the standard IACHEC model and that model if overplotted on the 2017 and 2018 data.

be 0.05. In this case, the center of the OBF-I is at $-55.8 \mathrm{C}$ and the center of the OBF-S is at $\sim-58.0 \mathrm{C}$. As contaminant accumulates on the filters and the surrounding surfaces the temperature distribution will change, with the centers of the filters becoming warmer. For an emittance of 0.20 , the center of the OBF-I increases to $-41.7 \mathrm{C}$ and the center of the OBF-S increases to $\sim-46.0 \mathrm{C}$. The temperatures of the OBFs increase as the emittance increases because the OBFs are more coupled to the temperature of the warm optical bench assembly $(+20 \mathrm{C})$. But as the emittance continues increasing the OBF temperatures start to decrease again because in this model, the surfaces around the OBF are also accumulating a contamination layer and those surfaces have a higher emittance which results in better coupling between those relatively cold surfaces and the OBFs.

As shown previously, ${ }^{18}$ the vaporization rate of materials is a steep function of temperature with the vaporization rate increasing by roughly one order of magnitude for every $5 \mathrm{C}$ increase in temperature. Therefore, it is possible that the vaporization rate has increased by about two to three orders of magnitude in the centers of the filters as the temperatures have increased from $\sim-56 \mathrm{C}$ to $\sim-42 \mathrm{C}$. . This could be part of the explanation for the reduction in the accumulation rate that has been observed. This would be consistent with the center of the OBF-I showing a larger reduction in the accumulation rate than the center of the OBF-S since the center of the OBF-I is warmer than the center of the OBF-S.

\section{FUTURE WORK}

The temporal model of the contamination correction in the N0010 file acisD1999-08-13contamN0010.fits contained in CALDB 4.7.8 needs modification to predict less absorption near the center and edges of the OBFs. This is clear from the E0102 line normalizations presented in Figures 7 and 9. The CXC calibration team is working on a revision to the N0010 model that will change the time dependence of the spatial distribution and will update the chemical composition as a function of time. We expect this revised contamination model to be released in two stages (both in 2018), one release for the OBF-I and one for OBF-S. 


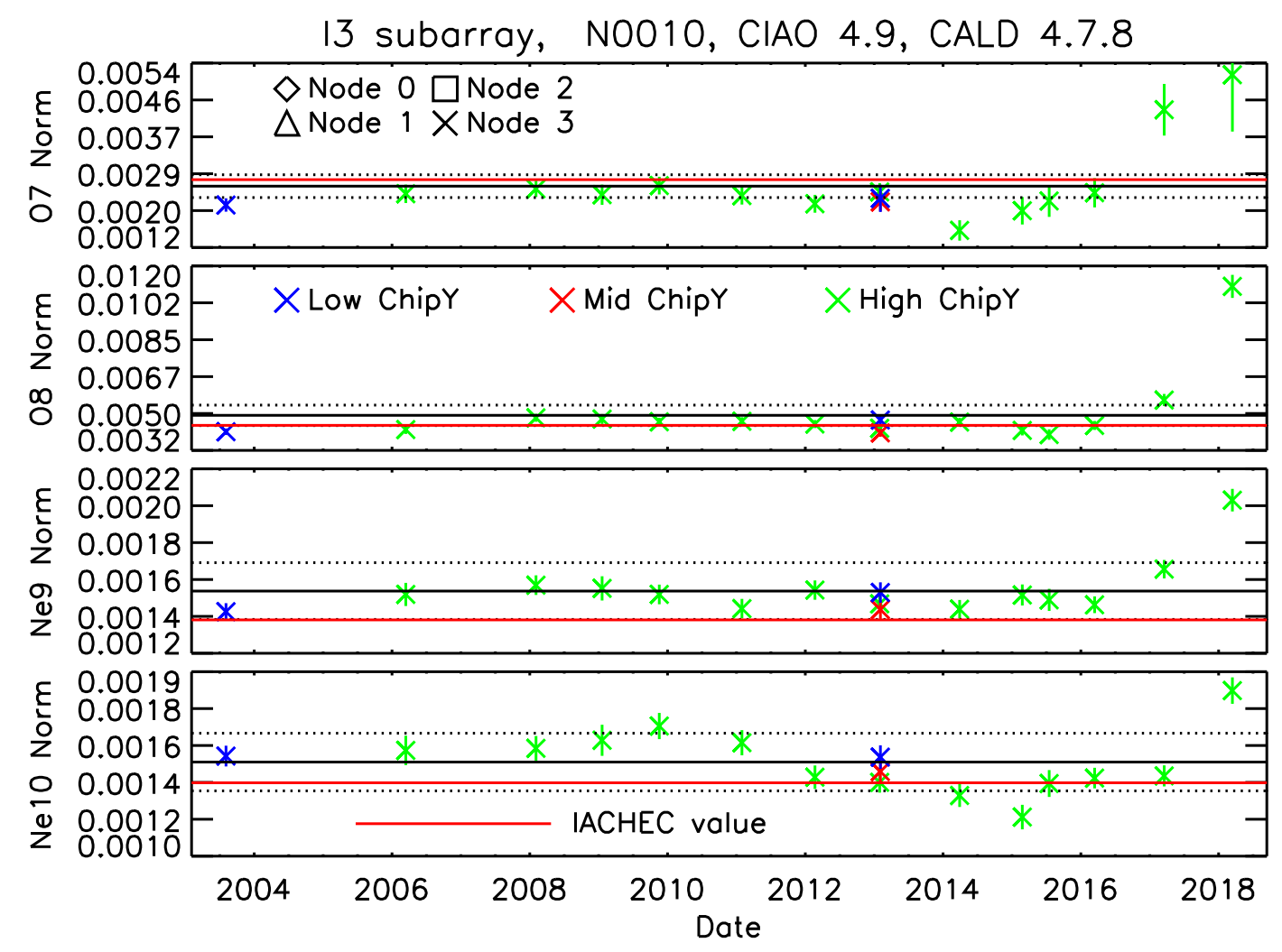

Figure 9. Line normalizations from E0102 on I3 as a function of time. The solid black line is the average of the data points near the on-axis point aimpoint. The red dashed lines are $+/-10 \%$ above and below the average. The points away from the nominal aimpoint are indicated in red and blue.

The accumulation rate of the contaminant will need to be monitored more frequently in the coming years. The accumulation rate at the centers and edges of the OBFs for both ACIS-S and ACIS-I have all changed in unexpected ways over the past two years. The continued characterization of these accumulation rates with time may provide constraints on the deposition and vaporization rates.

The CXO project considered a "Bakeout" of the ACIS instrument ${ }^{13}$ soon after the contamination layer was discovered in 2004. The project decided at that time that a Bakeout was not worth the risk. There have been several papers written describing models of an ACIS Bakeout, see O'Dell et al. (2005), ${ }^{19}$ O'Dell et al. (2013), ${ }^{18}$ and O'Dell et al. (2015). ${ }^{20}$ These papers predict a range of outcomes from successful to unsuccessful depending on the assumed volatilities for the contaminants. The recently discovered reduction in the accumulation rate makes it less likely the project will consider a Bakeout worth the risk. Nevertheless, we will continue to monitor the accumulation rate and spatial distribution of the contaminant to constrain the volatilities of the possible contaminants to hopefully to constrain the range of possible outcomes for a Bakeout. If the contaminant were observed to decrease in the center of the OBF-I and OBF-S, we would know that the vaporization rate is larger than the deposition rate at the current temperatures. Such a result would indicate that a Bakeout is likely to be successful, even at temperatures not much higher than the current range of - $70 \mathrm{C}$ to $-42 \mathrm{C}$.

\section{CONCLUSIONS}

We have presented the accumulation rate of the ACIS contamination layer as a function of time. The accumulation rate decreased from launch until 2005, was fairly linear from 2005 to 2010, increased after 2010 but has sharply decreased since 2016 . The chemical composition of the contamination has changed with time, possibly indicating that multiple sources are responsible for the contamination. The $\mathrm{C}, \mathrm{O}$, and $\mathrm{F}$ all exhibit different time dependencies again indicating that multiple materials have accumulated at different rates over the course 

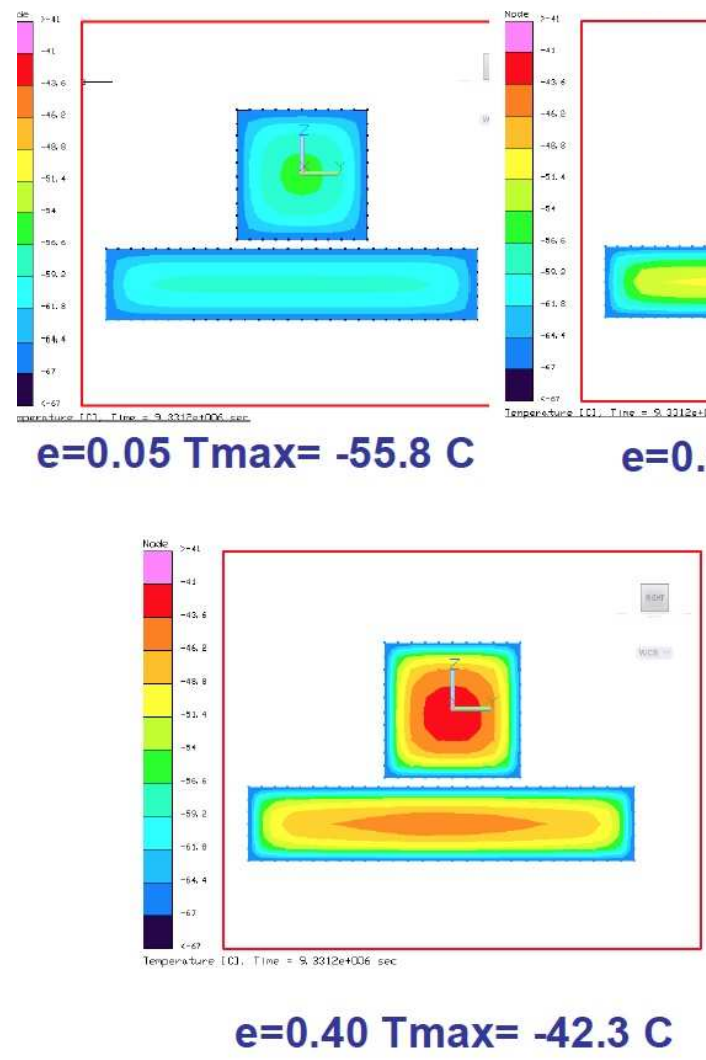

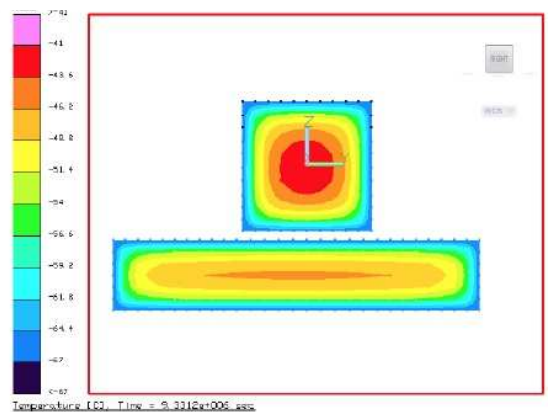

$\mathrm{e}=0.20 \mathrm{Tmax}=-41.7 \mathrm{C}$

$\mathrm{e}=0.10 \mathrm{Tmax}=-46.5 \mathrm{C}$

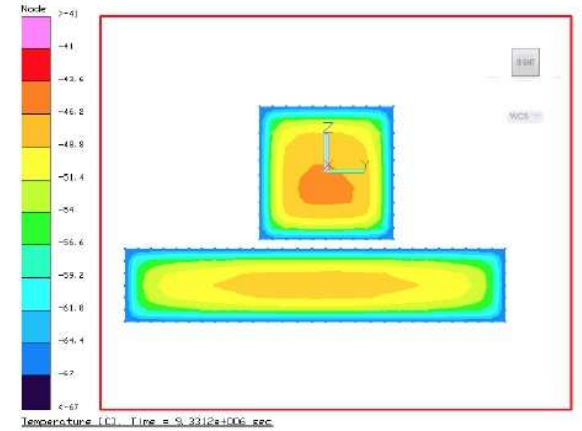

$\mathrm{e}=0.80 \mathrm{~T} \max =-45.6 \mathrm{C}$

Figure 10. The expected temperature distributions on the OBF-S and OBF-I as a function of emittance.

of the mission. Nevertheless, all three have shown a dramatic decrease over the past year. The explanation for this sudden decrease is not clear. It could be that the deposition rate has decreased or the vaporization rate has increased, or both. The CXC will need to monitor the contamination layer frequently with dedicated calibration observations over the coming years to accurately model the contamination layer

We tested the current contamination model N0010 with the SNR E0102. We find that the fitted values for the normalizations of the $\mathrm{O}$ VII He $\alpha r$ line, the $\mathrm{O}$ vin Ly $\alpha$ line, the Ne IX He $\alpha r$ line, and Nex Ly $\alpha$ line are mostly consistent to within $\pm 10 \%$ for both ACIS-S and ACIS-I near the aimpoint from 2003 through 2016. After 2016, the line normalizations begin to deviate from the average value, with deviations as large as $49 \%$ at the aimpoint on ACIS-S and $125 \%$ at the aimpoint on ACIS-I for the O VIII Ly $\alpha$ line. The CXC is preparing a revised contamination file that will significantly improve the agreement from 2016 onwards for release this year.

\section{ACKNOWLEDGMENTS}

We acknowledge support under NASA contract NAS8-03060. The Chandra X-ray Observatory is operated by the Smithsonian Astrophysical Observatory under contract to the NASA Marshall Space Flight Center (MSFC). The Advanced CCD Imaging Spectrometer (ACIS) was developed by the Massachusetts Institute of Technology and the Pennsylvania State University.

We sincerely thank all of our colleagues in the IACHEC that contributed to the development of the highly successful spectral model for E0102.

\section{REFERENCES}

[1] Weisskopf, M. C., Tananbaum, H. D., Van Speybroeck, L. P., and O'Dell, S. L., "Chandra x-ray observatory (cxo): overview," in [X-Ray Optics, Instruments, and Missions III], Truemper, J. E. and Aschenbach, B., eds., Proc. SPIE 4012, 2 (2000). 
[2] Weisskopf, M. C., "The Chandra X-Ray Observatory: progress report and highlights," in [Space Telescopes and Instrumentation 2012: Ultraviolet to Gamma Ray], Proc. SPIE 8443, 84430Y (Sept. 2012).

[3] Garmire, G., Ricker, G., Bautz, M., Burke, B., Burrows, D., Collins, S., Doty, J., Gendreau, K., Lumb, D., and Nousek, J., "The axaf ccd imaging spectrometer," in [American Institute of Aeronautics and Astronautics Conference], 8 (1992).

[4] Garmire, G. P., Bautz, M. W., Ford, P. G., Nousek, J. A., and Ricker, G. R., "Advanced CCD imaging spectrometer (ACIS) instrument on the Chandra X-ray Observatory," in [X-Ray and Gamma-Ray Telescopes and Instruments for Astronomy], Truemper, J. and Tananbaum, H., eds., Proc. SPIE 4851, 28-44 (Mar. 2003).

[5] Murray, S. S., Chappell, J. H., Kenter, A. T., Kobayashi, K., Kraft, R. P., Meehan, G. R., Zombeck, M. V., Fraser, G. W., Pearson, J. F., Lees, J. E., Brunton, A. N., Pearce, S. E., Barbera, M., Collura, A., and Serio, S., "AXAF High-Resolution Camera (HRC): calibration and recalibration at XRCF and beyond," in [EUV, X-Ray, and Gamma-Ray Instrumentation for Astronomy VIII], Siegmund, O. H. and Gummin, M. A., eds., Proc. SPIE 3114, 11-25 (Oct. 1997).

[6] Canizares, C. R., Huenemoerder, D. P., Davis, D. S., Dewey, D., Flanagan, K. A., Houck, J., Markert, T. H., Marshall, H. L., Schattenburg, M. L., Schulz, N. S., Wise, M., Drake, J. J., and Brickhouse, N. S., "High-Resolution X-Ray Spectra of Capella: Initial Results from the Chandra High-Energy Transmission Grating Spectrometer," Ap.J. 539, L41-L44 (Aug. 2000).

[7] Brinkman, A. C., "Description and Performance of the Low Energy Transmission Grating Spectrometer on-board Chandra," in [APS April Meeting Abstracts], (Apr. 2000).

[8] Plucinsky, P. P., Schulz, N. S., Marshall, H. L., Grant, C. E., Chartas, G., Sanwal, D., Teter, M., Vikhlinin, A. A., Edgar, R. J., Wise, M. W., Allen, G. E., Virani, S. N., DePasquale, J. M., and Raley, M. T., "Flight spectral response of the ACIS instrument," in [X-Ray and Gamma-Ray Telescopes and Instruments for Astronomy. Edited by Joachim E. Truemper, Harvey D. Tananbaum. Proceedings of the SPIE, Volume 4851, pp. 89-100 (2003).], Truemper, J. E. and Tananbaum, H. D., eds., 89-100 (Mar. 2003).

[9] Plucinsky, P. P., Bogdan, A., Germain, G., and Marshall, H. L., "The evolution of the ACIS contamination layer over the 16-year mission of the Chandra X-ray Observatory," in [Space Telescopes and Instrumentation 2016: Ultraviolet to Gamma Ray], Proc. SPIE 9905, 990544 (July 2016).

[10] Marshall, H. L., Tennant, A., Grant, C. E., Hitchcock, A. P., O’Dell, S. L., and Plucinsky, P. P., "Composition of the Chandra ACIS contaminant," in [X-Ray and Gamma-Ray Instrumentation for Astronomy XIII. Edited by Flanagan, Kathryn A.; Siegmund, Oswald H. W. Proceedings of the SPIE, Volume 5165, pp. 497-508 (2004).], Flanagan, K. A. and Siegmund, O. H. W., eds., 497-508 (Feb. 2004).

[11] Bautz, M., Pivovaroff, M., Baganoff, F., Isobe, T., Jones, S., Kissel, S., Lamarr, B., Manning, H., Prigozhin, G., Ricker, G., Nousek, J., Grant, C., Nishikida, K., Scholze, F., Thornagel, R., and Ulm, G., "X-ray ccd calibration for the axaf ccd imaging spectrometer," in [X-Ray Optics, Instruments, and Missions], Hoover, R. B. and II, A. B. W., eds., Proc. SPIE 3444, 210 (1998).

[12] Nousek, J. A., Townsley, L. K., Chartas, G., Burrows, D. N., Moskalenko, E., Sambruna, R., Pesce, J., Grant, C. E., Nishikida, K., Cawley, L. J., Broos, P. S., Koch, T. S., Garmire, A., Garmire, G. P., Bautz, M. W., Jones, S. E., Lamarr, B., and Ricker, G. R., "Joint AXAF high-resolution mirror assembly and AXAF CCD Imaging Spectrometer calibration at the MSFC X-Ray Calibration Facility," in [X-Ray Optics, Instruments, and Missions], Hoover, R. B. and Walker, A. B., eds., Proc. SPIE 3444, 225-233 (Nov. 1998).

[13] Plucinsky, P. P., O’Dell, S. L., Tice, N. W., Swartz, D. A., Bautz, M. W., DePasquale, J. M., Edgar, R. J., Garmire, G. P., Giordano, R., Grant, C. E., Knollenberg, P., Kissel, S., LaMarr, B., Logan, R., Mach, M., Marshall, H. L., McKendrick, L., Prigozhin, G. Y., Schwartz, D., Schulz, N. S., Shropshire, D., Trinh, T., Vikhlinin, A. A., and Virani, S. N., "An evaluation of a bake-out of the ACIS instrument on the Chandra X-Ray Observatory," in [Proceedings of the SPIE, Volume 5488, pp. 251-263 (2004).], Hasinger, G. and Turner, M. J. L., eds., 251-263 (Oct. 2004).

[14] Plucinsky, P. P., Haberl, F., Dewey, D., Beardmore, A. P., DePasquale, J. M., Godet, O., Grinberg, V., Miller, E. D., Pollock, A. M. T., Sembay, S., and Smith, R. K., "The SMC SNR 1E0102.2-7219 as a calibration standard for x-ray astronomy in the 0.3-2.5 keV bandpass," in [Society of Photo-Optical Instrumentation Engineers (SPIE) Conference Series], Society of Photo-Optical Instrumentation Engineers (SPIE) Conference Series $\mathbf{7 0 1 1}$ (Aug. 2008). 
[15] Plucinsky, P. P., Beardmore, A. P., DePasquale, J. M., Dewey, D., Foster, A., Haberl, F., Miller, E. D., Pollock, A. M. T., Posson-Brown, J. L. L., Sembay, S., and Smith, R. K., "Cross-calibration of the xray instruments onboard the Chandra, Suzaku, Swift, and XMM-Newton Observatories using the SNR 1E 0102.2-7219," in [Society of Photo-Optical Instrumentation Engineers (SPIE) Conference Series], Society of Photo-Optical Instrumentation Engineers (SPIE) Conference Series 8443 (Sept. 2012).

[16] Plucinsky, P. P., Beardmore, A. P., Foster, A., Haberl, F., Miller, E. D., Pollock, A. M. T., and Sembay, S., "SNR 1E 0102.2-7219 as an X-ray calibration standard in the 0.5-1.0 keV bandpass and its application to the CCD instruments aboard Chandra, Suzaku, Swift and XMM-Newton," Astronomy and Astrophysics 597, A35 (Jan. 2017).

[17] Smith, R. K., Brickhouse, N. S., Liedahl, D. A., and Raymond, J. C., "Collisional Plasma Models with APEC/APED: Emission-Line Diagnostics of Hydrogen-like and Helium-like Ions," ApJL 556, L91-L95 (Aug. 2001).

[18] O’Dell, S. L., Swartz, D. A., Tice, N. W., Plucinsky, P. P., Grant, C. E., Marshall, H. L., Vikhlinin, A., and Tennant, A. F., "Modeling contamination migration on the Chandra X-ray Observatory II," in [UV, X-Ray, and Gamma-Ray Space Instrumentation for Astronomy XVIII], Proc. SPIE 8859, 88590F (Sept. 2013).

[19] O’Dell, S. L., Swartz, D. A., Plucinsky, P. P., Freeman, M. A., Markevitch, M. L., Vikhlinin, A. A., Chen, K. C., Giordano, R. J., Knollenberg, P. J., Morris, P. A., Tran, H., Tice, N. W., and Anderson, S. K., "Modeling contamination migration on the Chandra X-ray Observatory," in [UV, X-Ray, and Gamma-Ray Space Instrumentation for Astronomy XIV], Siegmund, O. H. W., ed., Proc. SPIE 5898, 313-324 (Aug. 2005).

[20] O’Dell, S. L., Swartz, D. A., Tice, N. W., Plucinsky, P. P., Grant, C. E., Marshall, H. L., Vikhlinin, A. A., Tennant, A. F., and Dahmer, M. T., "Modeling contamination migration on the Chandra X-ray Observatory: III," in [UV, X-Ray, and Gamma-Ray Space Instrumentation for Astronomy XIX], Proc. SPIE 9601, 960107 (Sept. 2015). 